\title{
Diffuse Multifocal Bilateral Dysembryoplastic Neuroepithelial Tumor: A Very Unusual Case Report
}

\author{
Hassan Reza Mohammadi', Ehsan Moradi ${ }^{1}$, Elham Rahimian², Pascal Varlet ${ }^{3}{ }^{\circledR}$, Yalda Nilipour $^{4}{ }^{\circledR}$ \\ 'Department of Neurosurgery, Shahid Beheshti University of Medical Sciences, Tehran, Iran \\ ${ }^{2}$ Haghighat Research Center, Tehran, Iran \\ ${ }^{3}$ Department of Neuropathology, GHU Paris - Sainte-Anne Hospital, Paris, France \\ ${ }^{4}$ Pediatric Pathology Research Center, Research Institute for Children's Health, Shahid Beheshti University of Medical \\ Sciences, Tehran, Iran
}

\begin{abstract}
Dysembryoplastic neuroepithelial tumor (DNT) considered as a benign cortical glioneuronal neoplasm of children or young adults, typically present with drug-resistant focal epilepsy. DNTs are usually located in the temporal lobe but can found in any part of the supratentorial brain cortex. Multifocal DNTs have rarely reported. Here we present an eight years old boy with two years follow up, having a somewhat stable diffuse multinodular DNT of the most significant spatial extent that may have reported, involving cortical and subcortical left temporo-occipital lobe, bilateral basal ganglia and thalamus, presenting with headache, short stature, and behavioral disorder.

Keywords: Dysembryoplastic neuroepithelial tumor; Multifocal; Diffuse; Brain tumor; Bilateral
\end{abstract}

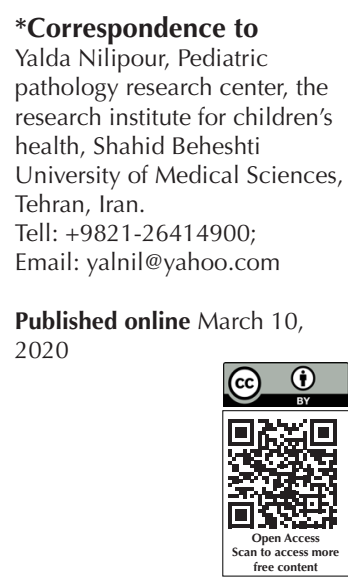

Citation: Mohammadi HR, Moradi E, Rahimian E, Varlet P, Nilipour Y. Diffuse Multifocal Bilateral Dysembryoplastic Neuroepithelia Tumor: A Very Unusual Case Report. Int Clin Neurosci J. 2020;7(2):109-112. doi:10.34172/icnj.2020.09.

\section{Introduction}

Dysembryoplastic neuroepithelial tumor (DNT) was first introduced as a new tumor entity by Professor Chaterine Daumas-Duport in $1988 .{ }^{1}$ DNT regarded as a WHO grade I tumor and is the second most common diagnosis of the specimens receiving from intractable epileptic surgeries. ${ }^{2}$ WHO classification of tumors of the central nervous system in 2016 defined DNT as "a benign Glioneuronal tumor usually located in the temporal lobe of children or young adults with drugresistant epilepsy; typically, with a cortical location and multinodular structure." DNTs have reported in any part of the supratentorial cortex, and only a few multifocal DNTs have reported in the English literature. Cortical location and showing neither mass effect nor significant peritumoral edema are essential criteria for the radiologic diagnosis of DNT. Three histologic subtypes of DNTs have described. The histopathological hallmark of DNT is a specific Glioneuronal element characterized by columns of bundles of axons lined by small oligo-like cells oriented vertically to the cortical surface and embedded in a myxoid matrix containing floating neurons. ${ }^{3}$ Long-term follow-up of patients having DNT typically reveals no progression or recurrence, even in patients with subtotal surgical resection. Here we present an eight years old boy with two years follows up, having a somewhat stable diffuse multinodular DNT of the most significant spatial extent that may have reported.

\section{Case Report}

An 8-year-old Iranian boy is presenting with short stature, headache, and recent behavioral changes as using foul language. He had no complained of seizure at that time. Brain MRI revealed multiple non-enhancing cortical and subcortical cystic nodules in the left temporooccipital lobe, basal ganglia, and left thalamus. Right basal ganglia also involved but apparently in less extension comparing with the left side. These nodules were hypointense or nearly isointense on T1- weighted images and hyperintense on T2-weighted and FLAIR images. It has measured $7.7 \times 5.6$ $\mathrm{cm}$. No visible mass effect or perilesional edema was seen (Figure 1). An initial radiologic diagnosis of low-grade glioma proposed. During one and a half years of follow up of the patient, the tumor had no change in size and configuration, but one deep posterior temporal nodule showed a new irregular peripheral enhancement (Figure 2). Brain MRA and MRV were normal. He started to have tonic colonic seizures two months before planning the 


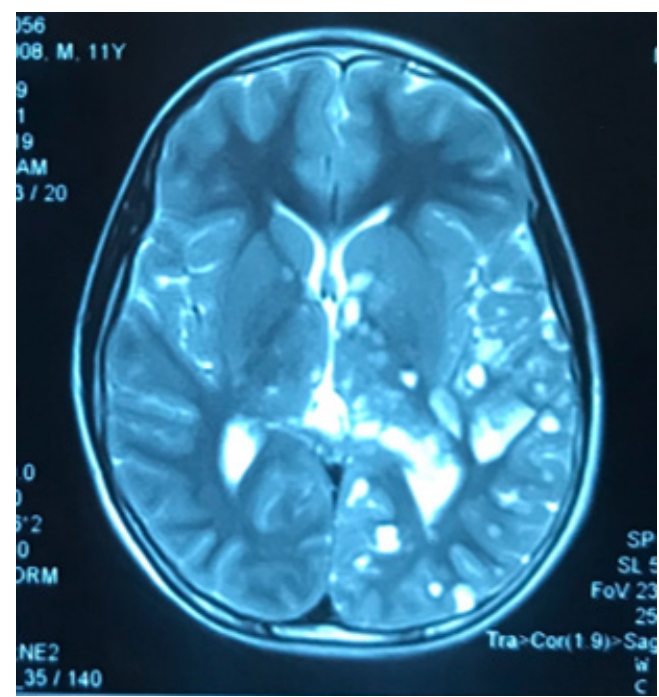

Figure 1. Brain MRI, Coronal, Hyper T2 Multiple Bilateral Nodular and Cystic Lesions of Left Temporal, Basal Ganglia, and Thalamus With No Considerable Mass Effect or Peripheral Edema.

lesional biopsy. Brain biopsy from the left temporal lobe performed. The histopathologic study revealed typical histologic features of DNT with nodules formation and typical specific Glioneuronal element with floating neurons (Figure 3 ) and also the linear superficial cortical spread of small oligo-like cells in a myxoid background (Figure 4). Neither mitosis nor necrosis has seen. The immunohistochemical study of Ki-67 was less than 1\%, and chromogranin stain highlights floating neurons. The diagnosis of diffuse multifocal dysembryoplastic neuroepithelial tumor was confirmed.

\section{Discussion}

Here we report a ten years old boy with diffuse cortical and subcortical cystic nodules of left temporal lobe also

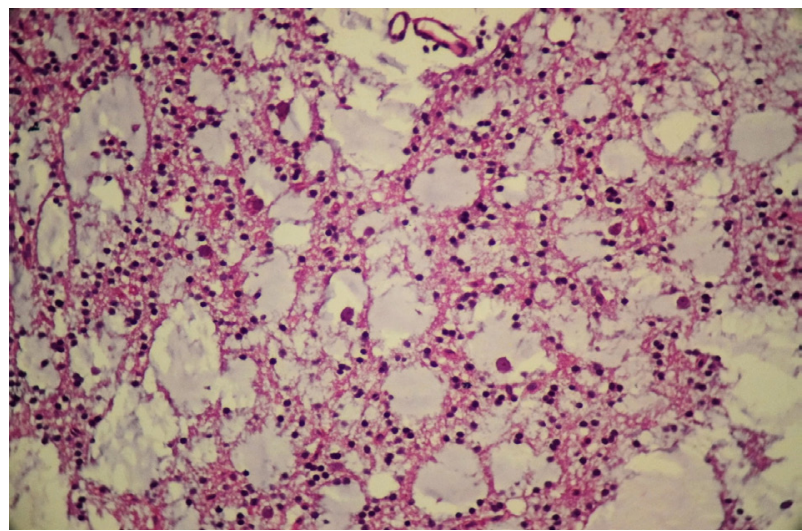

Figure 3. Column and Cysts Lined by Small Oligo-Like Cells in a Myxoid Background With the Presence of Floating Neurons (Specific Glioneuronal Element) (H\&E ×200).

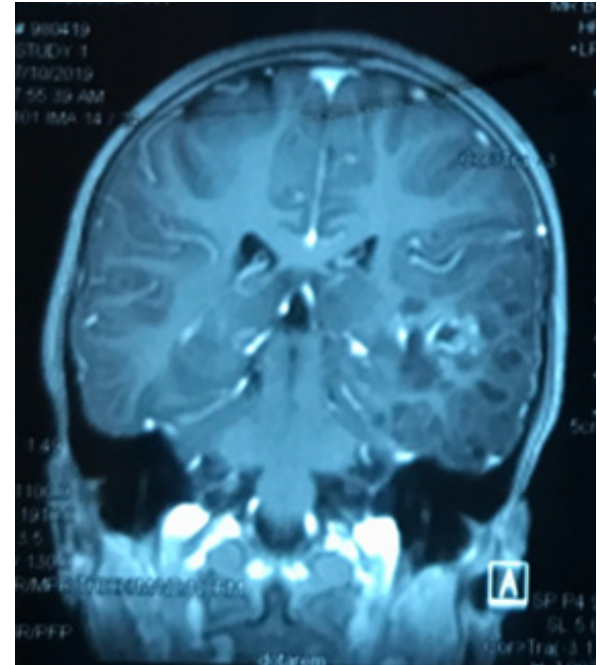

Figure 2. Brain MRI, Coronal, Multiple hypos- and Iso-signal T1 Lesions of Left Temporal Lobe With 1 Ring-Like Enhancement in Left Temporal.

involving occipital lobe, left thalamus, and bilateral basal ganglia, diagnosed as DNT by histopathology and immunohistochemical studies. DNTs can see in any part of the supratentorial cortex, but it has shown a strong predilection for the temporal lobe. It has also reported in the caudate nucleus, the lateral ventricles, the septum pellucidum, the trigonocephaly region, the midbrain, and the tectum, the third ventricle, the pons, the cerebellum, and brain stem. To the best of our knowledge, there are only seven reports of multifocal DNT in the English literature. . $^{3-10}$

The present case was mostly cortical with no prominent mass effect and almost unchanged in size and configuration in MRI studies during two years follow up. DNTs are usually cortical tumors with neither mass

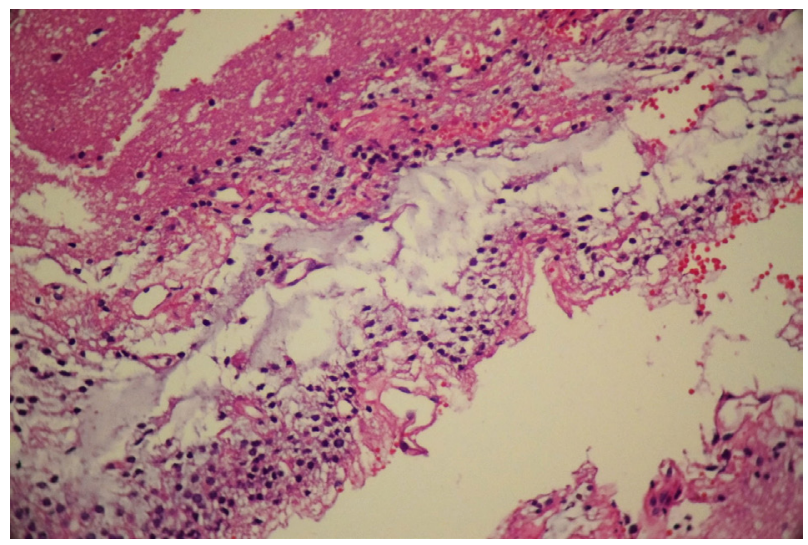

Figure 4. Linear Superficial Cortical Spread of Small Oligo-Like Cells in a Myxoid Background (H\&E ×200). 
effect nor significant peritumoral edema, and these are essential differentiating factors between DNTs and diffuse infiltrating gliomas. ${ }^{3} 20 \%$ to $35 \%$ of DNTs reveal contrast enhancement, usually as multiple rings. The present case developed a new ring-shaped enhancement in deep left temporal after about one and a half years, and since then, he started having seizures. Nodular or cystic contrast enhancement has reported in previously non-enhancing tumors. ${ }^{11}$ Changes in imaging follow-up in DNTs as increased tumor size, with or without peritumoral edema, have also been observed, although these changes have found not to be signs of malignant transformation and are usually due to ischemic and hemorrhagic events. ${ }^{4}$

There have defined three histologic variants of DNTs. The typical simple form is composed of only specific glioneuronal element and is devoid of other components. The complex form has multinodular architecture and is composed of glial nodules with astrocytic or oligodendrocytic differentiation with or without focal cortical dysplasia of the adjacent cortex, associated with specific glioneuronal element. The nonspecific or diffuse form of DNT has also described. Based on the lack of specific Glioneuronal element in this variant, it is difficult to distinguish this form from gangliogliomas or other gliomas by conventional histologic studies. ${ }^{2,3}$ The present case considered as a complex form with no focal cortical dysplasia in the received sample, and based on the presence of specific Glioneuronal element histologic and immunohistochemical diagnosis of this tumor was not a big challenge.

Daumas-Duport stated that DNTs has best classified as neoplasms based on the fact that they may form sizable mass-like lesions, induce clinical symptoms, and there is definite evidence of cell proliferation with possible areas of hypercellularity in some DNTs as well as the involvement of surrounding structures in some cases. There are also some reports of coexisting DNT with other low-grade gliomas in the literature as rosette-forming Glioneuronal tumor and Pilocytic astrocytoma. ${ }^{10}$ On the other hand, there is some evidence supporting that DNT is a hamartomatous or dysontogenic/malformative process. Young age of the patients, bone deformity adjacent to tumor in some cases, presence of adjacent cortical dysplasia in complex variants, the heterogeneous composition of some DNTs, deficient proliferative index activity in almost all variants, and benign clinical course of the patients in long term clinical follow-ups with patient's cure by surgical excision of the lesion are all evidences supporting the later theory. However, the exact histogenesis of DNT remains unknown.,13

Atypical clinical, radiologic, and histopathological findings must take into account for prediction of the biological behavior of DNT. A malignant transformation is an infrequent event, and it has reported after radiation and chemotherapy. ${ }^{3}$

\section{Genetic Profile}

Immunohistochemical studies and cytogenetic studies for fibroblast growth factor receptor 1 (FGFR1 ITD) and BRAF V600E mutations have been found in some instances of DNTs and prevent the risk of misdiagnosis. ${ }^{14}$ Diffuse gliomas markers such as IDH1 and K27M are absent in DNTs. ${ }^{3}$ Usually, DNTs have shown to have stable genomes, whereas recurrent gains of whole chromosomes 5 and 7 found in approximately $20 \%$ to $30 \%$ of DNT cases. It has stated that somatic FGFR1 alterations and MAP kinase pathway activation are the main events in the pathogenesis of DNET, and these findings are promising for the future coming targeted therapies. ${ }^{12,15}$

\section{References}

1. Daumas-Duport C, Scheithauer BW, Chodkiewicz JP, Laws ER Jr, Vedrenne C. Dysembryoplastic neuroepithelial tumor: a surgically curable tumor of young patients with intractable partial seizures. Report of thirty-nine cases. Neurosurgery. 1988;23(5):545-56. doi: 10.1227/00006123-19881100000002.

2. Suh YL. Dysembryoplastic neuroepithelial tumors. J Pathol Transl Med. 2015;49(6):438-49. doi: 10.4132/ jptm.2015.10.05.

3. Louis DN, Perry A, Reifenberger G, von Deimling A, FigarellaBranger D, Cavenee WK, et al. The 2016 World Health Organization classification of tumors of the central nervous system: a summary. Acta Neuropathol. 2016;131(6):803-20. doi: 10.1007/s00401-016-1545-1.

4. Sina F, Poranian M, Razmeh S, Taheri Talesh J. Multifocal dysembryoplastic neuroepithelial tumor: a report of a rare case. Rep Radiother Oncol. 2020; In Press. doi:10.5812/ rro.74048

5. Yang Al, Khawaja AM, Ballester-Fuentes L, Pack SD, Abdullaev Z, Patronas NJ, et al. Multifocal dysembryoplastic neuroepithelial tumours associated with refractory epilepsy. Epileptic Disord. 2014;16(3):328-32. doi: 10.1684/ epd.2014.0680.

6. Schittenhelm J, Mittelbronn $M$, Wolff $M$, Truebenbach J, Will BE, Meyermann R, et al. Multifocal dysembryoplastic neuroepithelial tumor with signs of atypia after regrowth. Neuropathology. 2007;27(4):383-9. doi: 10.1111/j.14401789.2007.00780.x.

7. Krossnes BK, Wester K, Moen G, Mørk SJ. Multifocal dysembryoplastic neuroepithelial tumour in a male with the XYY syndrome. Neuropathol Appl Neurobiol. 2005;31(5):55660. doi: 10.1111/j.1365-2990.2005.00680.x.

8. Whittle IR, Dow GR, Lammie GA, Wardlaw J. Dsyembryoplastic neuroepithelial tumour with discrete bilateral multifocality: further evidence for a germinal origin. Br J Neurosurg. 1999;13(5):508-11.

9. White RD, Kanodia AK, Sammler EM, Brunton JN, Heath CA. Multifocal dysembryoplastic neuroepithelial tumour with intradural spinal cord lipomas: report of a case. Case Rep Radiol. 2011;2011:734171. doi: 10.1155/2011/734171.

10. Lu JQ, Scheithauer BW, Sharma P, Scott JN, Parney IF, Hader W, et al. Multifocal complex glioneuronal tumor in an elderly man: an autopsy study: case report. Neurosurgery. 2009;64(6):E1193-5. doi: 10.1227/01. neu.0000345640.40566.48. 
11. Stanescu Cosson R, Varlet P, Beuvon F, Daumas Duport C, Devaux B, Chassoux F, et al. Dysembryoplastic neuroepithelial tumors: CT, MR findings and imaging follow-up: a study of 53 cases. J Neuroradiol. 2001;28(4):230-40.

12. Daumas-Duport C, Varlet $P$, Dysembryoplastic neuroepithelial tumors. Rev Neurol (Paris). 2003;59:622-36.

13. Gyure KA, Sandberg GD, Prayson RA, Morrison AL, Armstrong RC, Wong K. Dysembryoplastic neuroepithelial tumor: an immunohistochemical study with myelin oligodendrocyte glycoprotein. Arch Pathol Lab Med. 2000;124(1):123-6. doi: 10.1043/0003-9985(2000)124<0123:dnt>2.0.co;2.

14. Luzzi S, Elia A, Del Maestro M, Elbabaa SK, Carnevale S, Guerrini F, et al. Dysembryoplastic neuroepithelial tumors: what you need to know. World Neurosurg. 2019;127:25565. doi: 10.1016/j.wneu.2019.04.056.

15. Rivera B, Gayden T, Carrot-Zhang J, Nadaf J, Boshari T, Faury D, et al. Germline and somatic FGFR1 abnormalities in dysembryoplastic neuroepithelial tumors. Acta Neuropathol. 2016;131(6):847-63. doi: 10.1007/s00401-016-1549-x. 